\title{
Commentary: Tranexamic Acid in Patients Undergoing Coronary-Artery Surgery
}

\author{
Xiang-Dong $\mathrm{Wu}^{1}$, Ke-Jia $\mathrm{Hu}^{2,3}$ and Wei Huang ${ }^{1 *}$ \\ ${ }^{1}$ Department of Orthopaedic Surgery, The First Affiliated Hospital of Chongqing Medical University, Chongqing, China, \\ ${ }^{2}$ Department of Neurosurgery, Massachusetts General Hospital, Harvard Medical School, Boston, MA, United States, \\ ${ }^{3}$ Department of Microsurgery, Huashan Hospital, Fudan University, Shanghai, China
}

Keywords: tranexamic acid, seizures, thromboembolic complications, coronary-artery surgery, dose

\section{A commentary on}

Tranexamic Acid in Patients Undergoing Coronary-Artery Surgery

by Myles PS, Smith JA, Forbes A, Silbert B, Jayarajah M, Painter T, et al. N Engl J Med (2017) 376(2):136-48. doi: 10.1056/NEJMoa1606424

Tranexamic acid has been widely and routinely used as a prophylactic treatment to reduce the rate and severity of blood loss in many surgical disciplines, such as orthopedic and cardiac surgical procedures. However, whether such therapy would increase the risk of arterial or venous thrombotic events has long been uncertainty (1). Myles et al. performed the ATACAS trial published in NEJM, which underscored the benefits and potential risks of tranexamic acid in coronary-artery surgery (2). Tranexamic acid is associated with a lower risk of bleeding, without a higher risk of death or thrombotic complications, meanwhile, also higher postoperative seizures risk. This commentary aimed to discuss several issues related to tranexamic acid and the trial itself.

First, their work highlighted that the optimal dose and dosing regimens of tranexamic acid are still issues. On the one hand, their excellent work confirmed the efficacy of tranexamic acid to decrease blood loss, which is undisputed (3) and a definitive conclusion has been drawn from the literature (1). However, the increased incidence of seizures is not trivial. Indeed, seizures have been regarded as an adverse event of tranexamic acid for some time. Previous studies have confirmed a dosedependent relationship between the dose of tranexamic acid and incidence of seizures (4). A recent work compared the molecular structure of glycine with antifibrinolytic drugs and revealed a novel mechanism that tranexamic acid inhibits glycine receptors which explained why seizures increased after cardiovascular surgery (5). Accordingly, intravenous administration of high-dose tranexamic acid of $50 \mathrm{mg} / \mathrm{kg}$ and above should be deliberative, particularly in elder patients or patients who suffering from renal impairments (6). However, previous studies have indicated that high dose of tranexamic acid was more effective than low dose (7). It seems that we are trapped in a dilemma. Fortunately, multiple boluses of tranexamic acid have been proved to be superior to single boluse (8), hence, multiple boluses of low dose of tranexamic acid should be preferred and recommended as an alternative regimen to avoid tranexamic acid-induced seizures, and the optimal regimens warrant further investigation.

Second, Myles et al. considered that intravenous administration of tranexamic acid is not exempt from vascular accidents, the relationship of postoperative seizures with stroke and death observed in this trial suggests a possible underlying thromboembolic cause of the seizures. An observational study found that tranexamic acid was associated with an increased risk of venous thromboembolism, although the risk estimate did not reach statistical significance (9). Therefore, a growing tendency of thrombogenesis was strongly suspected even if no statistical 
significant differences were found regarding the risk of death or thrombotic complications. Noticeably, thrombotic event is extremely rare, thus question remains that no significant difference in thrombotic complications does not indicate no difference, or no changes in coagulability after intravenous of tranexamic acid.

Third, cardiac surgery would induce multifactorial changes in coagulability (10), while standard coagulation measurements have limited values as they just reflect deficiencies in procoagulant factors, without balancing concurrent deficiencies of anticoagulant factors such as thrombomodulin (11). Indeed, there has been a persistence of hypercoagulability state after cardiac surgery, and thromboelastography would be a preferable diagnostic assay to evaluate the accurate hemostasis and fibrinolysis (12). Additional perioperative coagulation assessment with thromboelastography might contribute to predict and detect the trend of the dynamic variation of coagulability, distinguish enzymatic hypercoagulability from platelet hypercoagulability or mixed hypercoagulability (13), which might not only reflect changes in coagulability and underlying prothrombotic tendency but also conducive to interpreting no significant differences in thrombotic events but in seizures. Regrettably, perioperative coagulation assessment was absent despite blood samples were collected (2). Marginal increase in hypercoagulability may not induce severe thrombotic complications, but perhaps relates to reduced cerebral blood flow and increased risk of cerebral infarction. One study reported ischemic strokes occurred after administration of tranexamic acid with a particular genotype, which indicated tranexamic acid may induce clinical seizures in susceptible patients (14) as a confounding factor.

One more concern is the primary outcome of the trial was a composite of death and a couple of thrombotic events. The investigators ignored the validity of composite end points. The present study incorporated component end points with heterogeneous importance to patients, the incidence of important components is small, and treatment effects differ significantly across components, which would be misleading when interpreting the results (15). If pulmonary embolism was the primary outcome, the required sample size would be more enormous; therefore, adverse events with low incidences were not ideal indicators.

\section{REFERENCES}

1. Ker K, Edwards P, Perel P, Shakur H, Roberts I. Effect of tranexamic acid on surgical bleeding: systematic review and cumulative meta-analysis. $B M J$ (2012) 344:e3054. doi:10.1136/bmj.e3054

2. Myles PS, Smith JA, Forbes A, Silbert B, Jayarajah M, Painter T, et al. Tranexamic acid in patients undergoing coronary-artery surgery. $N$ Engl J Med (2017) 376(2):136-48. doi:10.1056/NEJMoa1606424

3. Brown JR, Birkmeyer NJ, O'Connor GT. Meta-analysis comparing the effectiveness and adverse outcomes of antifibrinolytic agents in cardiac surgery. Circulation (2007) 115(22):2801-13. doi:10.1161/CIRCULATIONAHA.106.671222

4. Kalavrouziotis D, Voisine P, Mohammadi S, Dionne S, Dagenais F. Highdose tranexamic acid is an independent predictor of early seizure after cardiopulmonary bypass. Ann Thorac Surg (2012) 93(1):148-54. doi:10.1016/j. athoracsur.2011.07.085

5. Lecker I, Wang DS, Romaschin AD, Peterson M, Mazer CD, Orser BA. Tranexamic acid concentrations associated with human seizures inhibit glycine receptors. J Clin Invest (2012) 122(12):4654-66. doi:10.1172/JCI63375
In summary, current studies alerts and warns us that administration of high-dose of tranexamic acid needs discretion, multiple boluses of low-dose of tranexamic acid should be preferred and recommended as an alternative regimen in CABG, and future trials are warrant since no unanimous agreement reached and concerns remain regarding the optimal dosage and frequency of tranexamic acid. Tranexamic acid might induce hypercoagulability although no significant difference was detected in thrombotic complications, and thromboelastography would be a better detection method. The composite of the primary outcome in the results section of the trial is debatable. Whether these findings are relevant to other patient populations such as arthroplasty, requires further investigation.

\section{AUTHOR NOTES}

Guarantor of article: WH is acting as the submission's guarantor. Address: The First Affiliated Hospital of Chongqing Medical University, No. 1, Youyi Road, Yuanjiagang, Yuzhong District, Chongqing, 400016, China; Telephone number: (+86) 13883383330, Fax number: (+86) 023 89011212, E-mail: drhuangwei68@gmail.com. Submission statement: We confirm that this manuscript has not been published elsewhere and is not under consideration by another journal. All authors have approved the manuscript and agreed with submission to Frontiers in Cardiovascular Medicine.

\section{AUTHOR CONTRIBUTIONS}

$\mathrm{X}$-DW contributed substantially to conception and design and acquisition, analysis, and interpretation of data; drafted the article; gave final approval of the version to be published; and agreed to act as guarantor of the work. K-JH contributed substantially to acquisition, analysis, and interpretation of data; drafted the article; gave final approval of the version to be published; and agreed to act as guarantor of the work. WH contributed substantially to conception and design and acquisition, analysis, and interpretation of data; revised it critically for important intellectual content; gave final approval of the version to be published; and agreed to act as guarantor of the work.

6. Keyl C, Uhl R, Beyersdorf F, Stampf S, Lehane C, Wiesenack C, et al. Highdose tranexamic acid is related to increased risk of generalized seizures after aortic valve replacement. Eur J Cardiothorac Surg (2011) 39(5):e114-21. doi:10.1016/j.ejcts.2010.12.030

7. Sigaut S, Tremey B, Ouattara A, Couturier R, Taberlet C, Grassin-Delyle S, et al. Comparison of two doses of tranexamic acid in adults undergoing cardiac surgery with cardiopulmonary bypass. Anesthesiology (2014) 120(3):590-600. doi:10.1097/ALN.0b013e3182a443e8

8. Xie J, Ma J, Yao H, Yue C, Pei F. Multiple boluses of intravenous tranexamic acid to reduce hidden blood loss after primary total knee arthroplasty without tourniquet: a randomized clinical trial. J Arthroplasty (2016) 31(11):2458-64. doi:10.1016/j.arth.2016.04.034

9. Sundstrom A, Seaman H, Kieler H, Alfredsson L. The risk of venous thromboembolism associated with the use of tranexamic acid and other drugs used to treat menorrhagia: a case-control study using the general practice research database. BJOG (2009) 116(1):91-7. doi:10.1111/j.1471-0528.2008.01926.x

10. Tang M, Wierup P, Rea CJ, Ingerslev J, Hjortdal VE, Srensen B. Temporal changes in clot lysis and clot stability following tranexamic acid in cardiac 
surgery. Blood Coagul Fibrinolysis (2017) 28(4):295-302. doi:10.1097/ MBC. 0000000000000595

11. Tripodi A, Caldwell SH, Hoffman M, Trotter JF, Sanyal AJ. Review article: the prothrombin time test as a measure of bleeding risk and prognosis in liver disease. Aliment Pharmacol Ther (2007) 26(2):141-8. doi:10.1111/ j.1365-2036.2007.03369.x

12. Salooja N, Perry DJ. Thrombelastography. Blood Coagul Fibrinolysis (2001) 12(5):327-37. doi:10.1097/00001721-200107000-00001

13. Gonzalez E, Kashuk JL, Moore EE, Silliman CC. Differentiation of enzymatic from platelet hypercoagulability using the novel thrombelastography parameter delta (delta). J Surg Res (2010) 163(1):96-101. doi:10.1016/j.jss. 2010.03.058

14. Nardi K, Pelone G, Bartolo M, Di Ruzza MR, Storto M, Notte A, et al. Ischaemic stroke following tranexamic acid in young patients carrying heterozygosity of MTHFR C677T. Ann Clin Biochem (2011) 48(Pt 6):575-8. doi:10.1258/ acb.2011.011101
15. Ferreira-Gonzalez I, Busse JW, Heels-Ansdell D, Montori VM, Akl EA, Bryant DM, et al. Problems with use of composite end points in cardiovascular trials: systematic review of randomised controlled trials. BMJ (2007) 334(7597):786. doi:10.1136/bmj.39136.682083.AE

Conflict of Interest Statement: The authors declare that the research was conducted in the absence of any commercial or financial relationships that could be construed as a potential conflict of interest.

Copyright $\odot 2017 \mathrm{Wu}, \mathrm{Hu}$ and Huang. This is an open-access article distributed under the terms of the Creative Commons Attribution License (CC BY). The use, distribution or reproduction in other forums is permitted, provided the original author(s) or licensor are credited and that the original publication in this journal is cited, in accordance with accepted academic practice. No use, distribution or reproduction is permitted which does not comply with these terms. 